ISSN (print) 0867-2008 / ISSN (online) 239 1-75 12

DOI: http://dx.doi.org/10.12775/OM.2021.005

PřemYSL BAR*

Ústav pomocných věd historických a archivnictví

Filozofická fakulta

Masarykova univerzita

Arna Nováka 1

Cz-Brno 60200

Czech Republic

bar@phil.muni.cz

\title{
ÜBER EINIGE ASPEKTE DES DIPLOMATISCHEN VERKEHRS ZWISCHEN DEM HOCHMEISTER UND KAISER SIGISMUND VON LUXEMBURG**
}

\section{KEYWORDS}

bistory; the Middle Ages; military orders; Teutonic Order; diplomatic traffic; legations; correspondence; Sigismund of Luxembourg

\section{ABSTRACT \\ On some aspects of the diplomatic traffic between the Grand Master of the Teutonic Order and Emperor Sigismund of Luxembourg}

The diplomatic traffic between the Grand Master of the Teutnic Order and Emperor Sigismund of Luxembourg was carried out according to the common practices of diplomacy in late medieval Europe. Nevertheless, this topic deserves further exploration due to Sigismund's efforts to impose suzerainty upon the Grand Master and the Teutonic Knights. This issue influenced their mutual relations after Sigismund's election as Roman-German King in $1410 / 1411$.

There are numerous surviving sources, especially in the archive of the Teutonic Order in Berlin (GStA $\mathrm{PK})$, such as legation's instruction, dispatches and, last but not least, the political correspondence between the Grand Master and Emperor Sigismund. These sources can shed light not only on the complicated diplomatic

* ORCID: https://orcid.org/0000-000 I-9579-4509

** Diese Studie wurde von der Tschechischen Forschungsgemeinschaft (GA ČR) im Rahmen des Projekts EXPRO 19-28415X „From Performativity to Institutionalization: Handling Conflict in the Late Middle Ages (Strategies, Agents, Communication) “ gefördert. Sie entstand an der Philosophischen Fakultät der Masaryk-Universität. 
relation between above-mentioned two entities, but also, due to richness of their content, on late medieval diplomacy in general.

Based upon the research findings by Klaus Neitmann, who explored the Order's legation exclusively, this paper tries to expand the field of research by including the legations of Sigismund. From this perspective only several selected aspects of the topic are examined in the study: I) defining a legation (foreign mission) and its characteristic features; 2 ) the diplomatic traffic between the Grand Master and Sigismund of Luxembourg from a prosopographical perspective; and 3 ) the personal composition and communication at the court of Sigismund. The richness of sources makes new questions possible concerning not only this specific diplomatic traffic, but also late medieval diplomacy in general as well. However, the definite answers might be delivered after compiling a thorough list of all legations from both sides, which in light of the large number of primary sources must be reserved for another study.

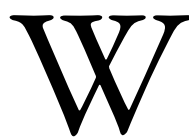

ill man den diplomatischen Verkehr zwischen dem Hochmeister und Sigismund von Luxemburg behandeln, muss zunächst die grundlegende Frage gestellt werden, ob es sich überhaupt lohnt, gerade diesem Thema besondere Aufmerksamkeit zu widmen. Man kann nämlich davon ausgehen, dass der hier behandelte diplomatische Verkehr sich kaum von der allgemeinen spätmittelalterlichen diplomatischen Tätigkeit unterscheidet, wie sie z.B. in den Arbeiten von Arnd Reitemeier ${ }^{1}$ oder Martin Kintzinger ${ }^{2}$ dargestellt wird. Mindestens aus zwei Gründen scheint es gerechtfertigt, sich mit dem Thema zu befassen. Es geht nämlich nicht allein darum, neue Fakten zum Gesandtschaftswesen im Spätmittelalter zusammenzutragen. Das Fallbeispiel selbst kann etwas Neues zu dem allgemeinen Phänomen der spätmittelalterlichen Diplomatie beitragen.

In zweierlei Hinsicht kann der diplomatische Betrieb zwischen Kaiser Sigismund und dem Hochmeister beachtliches Forschungspotenzial bergen. Erstens ist es die relativ gute Überlieferungssituation der jeweiligen diplomatischen Quellen, besonders wenn man an das Ordensarchiv im Geheimen Staatsarchiv Preußischer Kulturbesitz (GStA PK) in Berlin denkt. ${ }^{3}$ Zweitens ist die Behandlung des diplomatischen Verkehrs im Hinblick auf das ambivalente Verhältnis zwischen König Sigismund und dem Hochmeister beachtenswert. Allgemein kann man diese

I Arnd Reitemeier, Außenpolitik im Spätmittelalter. Die diplomatischen Beziehungen zwischen dem Reich und England 1377-1422 (Paderborn-München-Wien-Zürich: Schöningh, 1999).

2 Martin Kintzinger, Westbindungen im spätmittelalterlichen Europa. Auswärtige Politik zwischen dem Reich, Frankreich, Burgund und England in der Regierungszeit Kaiser Sigmunds (Stuttgart: Thorbecke, 2000).

3 Aus dieser Tatsache ergibt sich jedoch nicht, dass die diplomatischen Quellen für gesamte Zeitperiode sowie für beide Seiten jener politischen Beziehung gleichmäßig überliefert sind. Im Allgemeinen kann man feststellen, dass die Quellen aus der Provenienz des Deutschen Ordens in einem größeren Umfang und besseren Zustand erhalten sind. 
Beziehungen Sigismunds zum Hochmeister und zum Deutschen Orden als pragmatisch und deswegen wechselhaft bezeichnen. ${ }^{4}$

Bis zum sog. „Großen Krieg“ I 409-I 4 I I war Sigismund als König von Ungarn der treue Verbündete und Unterstützer des Deutschen Ordens. ${ }^{5}$ Nach der herben Niederlage des Ordens bei Tannenberg ( I 5. Juli I 4 I O) näherte sich Sigismund dem König von Polen (Władysław II. Jagiełło) an und schloss mit diesem am I 5. März I 4 I 2 ein Bündnis ab (sog. Vertrag von Lublau), ${ }^{6}$ das jedoch gegebenenfalls ein ähnliches Bündnis mit dem Deutschen Orden nicht ausschließen musste. ${ }^{7}$ Sigismund wollte offensichtlich beide Mächte, also das Königreich Polen und den Deutschen Orden, durch das ihm zugesprochene Schiedsrichteramt so fest wie möglich an sich und seine politischen Projekte binden. ${ }^{8}$

Nachdem der Hochmeister die Bedingungen des von Sigismund angebotenen Bündnisses abgelehnt hatte, ${ }^{9}$ versuchte der römische König den Hochmeister dazu zu bewegen, die Lehnsoberhoheit des römisch-deutschen Reichs zu akzeptieren, ${ }^{10}$ jedoch vergeblich. Der Hochmeister weigerte sich, diese ultimative Forderung anzunehmen, bis sich die Situation im Jahre 14 I 9 so zuspitzte, dass König Sigismund dem Orden öffentlich androhte, dessen Territorium gemeinsam mit Polen

4 Ottokar Israel, Das Verhältnis des Hochmeisters des Deutschen Ordens zum Reich im I5. Jahrhundert (Marburg: Johann Gottfried Herder Institut, I 952), 3-4 I; Jörg K. Hoensch, "König/ /Kaiser Sigismund, der Deutsche Orden und Polen-Litauen. Stationen einer problembeladenen Beziehung," Zeitschrift für Ostmitteleuropa-Forschung 46 (I 997): I-44.

5 Zenon H. Nowak, Polityka pótnocna Zygmunta Luksemburskiego do roku I4II, Roczniki Towarzystwa Naukowego w Toruniu 69/ I (Toruń: Państwowe Wydawnictwo Naukowe, 1964).

6 Die Staatsverträge des Deutschen Ordens in Preussen im Is. Jahrhundert, Bd. I (1398-1437), hrsg. v. Erich Weise (Marburg: N. G. Elwert, I 970), Nr. 90.

7 Ebd., Nrn. 86-87.

8 Vgl. Zenon H. Nowak, Międzynarodowe procesy polubowne jako narzędzie polityki Zygmunta Luksemburskiego w pótnocnej i środkowowschodniej Europie (I4I2-I424) (Toruń: Uniwersytet Mikołaja Kopernika, I98 I), I 3-47, 6 I-I 23; Přemysl Bar, "A Tortuous Path to Reconciliation and Justice. Sigismund of Luxembourg as Arbiter in the Dispute between the Teutonic Knights and Poland (1 4 I 2-1 420)," Zeitschrift für Ostmitteleuropa-Forschung 66 (2017): 3-40.

9 In der Forschung wird behauptet, dass der Hochmeister den Bündnisvertrag mit König Sigismund nicht ratifiziert habe, siehe Wilhelm Nöbel, Michael Küchmeister. Hochmeister des Deutschen Ordens 1414-1422, Quellen und Studien zur Geschichte des Deutschen Ordens 5 (Bad Godesberg: Verlag Wissenschaftliches Archiv, 1969), 46-49; Hoensch, "König/Kaiser Sigismund," I 3 - I 4 .

10 Die Beziehung König Sigismunds zum Hochmeister ist der Bestandteil größerer Problematik des Verhältnisses des Deutschen Ordens zum deutsch-römischen Reich, was hier nicht erörtert werden kann, vgl. Ingrid Matison, "Die Lehnsexemtion des Deutschen Ordens und dessen staatsrechtliche Stellung in Preußen," Deutsches Archiv für Erforschung des Mittelalters 2 I (1965): 194-248, wo weitere Literaturhinweise zum Thema vorhanden sind. 
anzugreifen. ${ }^{11}$ Durch den zugunsten des Ordens verkündeten Breslauer Schiedsspruch drehte jedoch die gesamte politische Konstellation um I 80 Grad. ${ }^{12}$ Das nun angespannte Verhältnis zwischen Sigismund und Polen wurde relativ schnell ausgeglichen, was im Abschluss eines Bündnisses im März 1423 gipfelte. ${ }^{13}$ Auch damals bot Sigismund dem Hochmeister ein ähnliches Bündnis, was wieder abgelehnt wurde. ${ }^{14}$

In der zweiten Hälfte der zoer Jahren verschlechterten sich die Beziehungen zwischen Sigismund und dem König von Polen allmählich wieder, vor allem wegen der polnischen Eingriffe in die Verhandlungen mit den Hussiten, was Sigismund als ungehörig im Hinblick auf seine Prärogativen als König von Böhmen empfunden haben muss. ${ }^{15}$ Sigismund versuchte, diese Tendenz durch ein Bündnis mit Litauen und dem Hochmeister zu verhindern. Dies wurde deutlich, als er bei dem Herrschertreffen in Luck (heute Luck' in der nordwestlichen Ukraine) im Januar I 429 dem litauischen Großfürsten Witold die königliche Krone (nicht zum ersten Mal) und die Erhebung Litauens zum Königtum offerierte. Diesen politischen Plan, nämlich eine Allianz der verbündeten Königreiche einschließlich des Ordensstaates gegen Polen zu schaffen, realisierte Sigismund auch nach dem Tod Witolds im Oktober I 430. ${ }^{16}$ Deswegen war er grundsätzlich gegen die politische Entwicklung, welche im Abschluss des ewigen Friedens (des sog. Friedens von Brest) zwischen dem Orden und Polen im Jahr I 435 gipfelte. ${ }^{17}$

in Komtur von Thorn Ulrich Zenger schildert in seinem Bericht (24. Mai I 419, Kaschau) zweideutige Stellungnahme König Sigismunds zum Hochmeister und dem Orden, indem einerseits der König über das treue Verhalten des Ordens zu ihm im vertraulichen Gespräch sich überzeugen konnte, aber anderseits öffentlich über militärische Hilfeleistung für Władysław II. Jagiełło rede, siehe Berlin, Geheimes Staatsarchiv Preußischer Kulturbesitz (weiterhin als: GStA PK), XX. Hauptabteilung (weiterhin als: HA), Ordensbriefarchiv (weiterhin als: OBA), Nr. 2969; Teildruck siehe bei Israel, Das Verbältnis, 95 Nr. 20.

12 Die Staatsverträge, I, hrsg. v. Weise, Nr. 14 I.

13 Regesta Imperii XI. Die Urkunden Kaiser Sigmunds (I4IO-I437) (weiterhin als: RI, XI), Bd. I-2 (I4IO-I437), hrsg. v. Wilhelm Altmann (Innsbruck: Verlag der Wagner'schen UniversitätsBuchhandlung, I 896-1900), Nr. 5493.

14 Carl A. Lückerath, Paul von Rusdorf. Hochmeister des Deutschen Ordens, 1422-I44I, Quellen und Studien zur Geschichte des Deutschen Ordens is (Bad Godesberg: Verlag Wissenschaftliches Archiv, I 969), 78-79.

is Jarosław Nikodem, Polska i Litwa wobec husyckich Czech w latach 1420-1433. Studium o polityce dynastycznej Wtadystawa Jagietty i Witolda Kiejstutowicza (Oświęcim: Napoleon V, 2015 , 2. Auflage).

16 Přemysl Bar, “Der 'Krönungssturm' König Sigismund von Luxemburg, Großfürst Witold von Litauen und das gescheiterte politische Bündnis zwischen beiden Herrschern," Roczniki Historyczne 83 (2017): 65-101.

17 Lückerath, Paul von Rusdorf, 169-172. 
Selbst dieser nur knappe Überblick von der sich immer wandelnden Beziehungen zwischen König/Kaiser Sigismund und dem Hochmeister hilft zu begreifen, dass die beiderseitigen Gesandtschaften zwischen Sigismund und dem Hochmeister eine eingehendere Untersuchung verdienen. Es ist davon auszugehen, dass die Gesandtschaften diese Beziehungen nicht nur widergespiegelt, sondern auch mitgeprägt haben.

Dementsprechend scheint das Vorhaben, alle wechselseitigen Gesandtschaften zwischen dem Hochmeister und Sigismund von Luxemburg aufgrund der jeweiligen vorhandenen Quellen zusammenzustellen, dann das gesammelte Material statistisch und interpretativ auszuwerten und schließlich neue Forschungsfragen $\mathrm{zu}$ formulieren, nutzbringend zu sein. Eine solche vollständige Liste beiderseitiger Gesandtschaften sollte chronologisch den Zeitraum ab i 378, als Sigismund zehnjährig Markgraf von Brandenburg wurde, bis dessen Tod, also bis zum 9. Dezember I 437, umfassen. Wegen deren Umfang kann jedoch kein vollständiges Verzeichnis vorgelegt, sondern es können lediglich einige Aspekte zu dem hier vorliegenden Thema veröffentlicht werden.

Als Ausgangsbasis muss die Zusammenstellung aller Ordensgesandtschaften dienen, die Klaus Neitmann in seinem Buch zu den Staatsverträgen des Deutschen Ordens vorgenommen hat. Sein Verzeichnis, das alle Ordensgesandtschaften aus den Jahren I 382-I 449 umfasst, entstand aufgrund der vorhandenen Editionen und teilweise des Ordensbriefarchivs in GStA PK. ${ }^{18}$ So hat Neitmann 37, bzw. 36 Ordensgesandtschaften zu Sigismund verzeichnet. ${ }^{19}$ Dazu sollen aufgrund neuer Recherchen weitere 26 Ordensgesandtschaften hinzugefügt werden. Zusammen macht dies 63, bzw. 62 Ordensgesandtschaften zu Sigismund. Das Gleiche gilt für die bisher in der Forschung nur teilweise berücksichtigten Gesandtschaften König Sigismunds, deren Zahl etwas niedriger, nämlich bei 5 I, liegt. ${ }^{20}$

18 Klaus Neitmann, Die Staatsverträge des Deutschen Ordens in Preußen 1230-1449. Studien zur Diplomatie eines spätmittelalterlichen deutschen Territorialstaates (Köln-Wien: Böhlau Verlag, 1986), 619-638.

19 Eine Gesandtschaft aus dem Verzeichnis Neitmanns soll nämlich weggelassen werden. Im Juni I 4 I 8 wurde Ludwig von Landsee, Komtur von Thorn, damit beauftragt, die drei Ordensdörfer Orlau, Morin und Neuendorf den Vertretern König Sigismunds zu überantworten. Er zog nach Ungarn jedoch nicht, sondern erwartete die königlichen Gesandten in Thorn, vgl. Archiwum Państwowe w Gdańsku, 300, R/Ll, Nr. 74, S. 36-38 (= Fol. 24b-25b) (Abschrift aus dem verschollenen Ordensfoliant I o, Nr. 43); Neitmann, Die Staatsverträge, 628 Nr. 173 ; Klaus Neitmann, "Ludwig von Landsee, ein Gebietiger des Deutschen Ordens in Preußen im I 5. Jahrhundert. Beobachtungen zur Außenpolitik des Ordens," Jahrbücher für Geschichte Osteuropas N.F. 36 (1988): 164.

20 Die Zahlen sollen jedoch als ungefähr und vorläufig betrachtet werden, bis jeweiliges Verzeichnis der Gesandtschaften fertig gestellt wird. 
Die Vermehrung der Anzahl der Gesandtschaften wird durch unentbehrliche und anspruchsvolle heuristische Arbeit sowie die Archivrecherchen ermöglicht. Es geht dabei nicht nur um eine Suche nach Schriftstücken der Diplomatie, wie Kredenzbriefen (littera credencia), Vollmachten (littera procuratoria), Geleitbriefen (salvus conductus), Instruktionen oder Berichten, sondern auch um die wechselseitige Korrespondenz, ${ }^{21}$ die die verschiedenen Aspekte der Gesandtschaften beleuchten und sogar eine Information über sonst eine unbekannte Gesandtschaft bieten kann. Nicht zuletzt darf man keine chronikalische Überlieferung oder auch die Rechnungsquellen beiseitelassen, wie z.B. das „Marienburger Tresslerbuch.“.22

Die Überlieferung der genannten Quellengattungen sowie ihre Erschließung ist nicht nur unvollständig, sondern auch ungleichmäßig verteilt. Ein Beispiel können die Berichte darstellen, die die Gesandten ihren Auftraggebern erstattet haben. Unter den Ordensgesandtschaften kann man etwa 25 Gesandtschaften errechnen, zu denen ein oder mehrere Berichte erhalten sind, im Vergleich zu drei Berichten (!) der königlichen Gesandtschaft. ${ }^{23}$

Demgegenüber ist die Korrespondenz zwar besser erschlossen, aber dennoch unvollständig. Während die Briefe König Sigismunds für die Hochmeister (es handelt sich um i2s Stücke) im Rahmen der Regesta Imperii, entweder von Wilhelm Altmann oder aufgrund der Neubearbeitung (bislang drei Bände), relativ gut erforscht sind, ${ }^{24}$ sind die Briefe der Hochmeister nur teilweise zugänglich.

${ }_{21}$ Vgl. Reitemeier, Außenpolitik, 35-63.

22 Das Marienburger Tresslerbuch der Jahre 1399-1409, hrsg. v. Erich Joachim (Königsberg i. Pr.: Thomas \& Oppermann, ı 896); vgl. Hartmut Boockmann, "Nachrichten über die Diplomatie des Deutschen Ordens aus dem 'Tresslerbuch'," in Balticum. Studia z dziejów polityki, gospodarki i kultury XII-XVII wieku ofiarowane Marianowi Biskupowi w siedemdziesiata rocznice urodzin, hrsg. v. Zenon H. Nowak (Toruń: Towarzystwo Naukowe w Toruniu, I992), 67-78.

23 Es handelt sich unter anderem um die Gesandtschaft des Herzogs Přemek von Troppau und des königlichen Hofmeisters Ludwig von Ottingen vom Mai bis Juni I 4 I 9. Ihr Bericht (1 3. Juni I 4 I 9, Thorn) wurde ursprünglich als eine Beilage dem Brief König Sigismunds ( I 8. Juni I 4 I 9 , Kaschau) an einen ungenannten schlesischen Herzog hinzugefügt. Heute sind beide Stücke als zeitnahe Abschrift in GStA PK, XX. HA, OBA, Nr. 2977 aufbewahrt.

24 Das Material wird aber nur aus der römischen Regierungszeit Sigismunds bearbeitet, also ab I 410/14I I bis 1437, siehe Anm. I 3 und Regesta Imperii XI. Regesten Kaiser Sigismunds (1410-1437) nach Archiven und Bibliotheken geordnet, Bd. I, Die Urkunden und Briefe aus den Archiven und Bibliotheken Mährens und Tschechisch-Schlesiens, nach Wilhelm Altmann neubearb. v. Peter Elbel (Wien-Köln-Weimar: Böhlau, 201 2); Regesta Imperii XI. Regesten Kaiser Sigismunds (1410-1437) nach Archiven und Bibliotheken geordnet, Bd. 2, Die Urkunden und Briefe aus den Archiven und Bibliotheken West-, Nord-und Ostböhmens, nach Wilhelm Altmann neubearb. v. Petr Elbel, Stanislav Bárta, Přemysl Bar, und Lukáš Reitinger (Wien-Köln-Weimar: Böhlau Verlag, 201 5); Regesta Imperii XI. Regesten Kaiser Sigismunds (14I0-I437) nach Archiven und Bibliotheken geordnet, Bd. 3, Die Urkunden aus den Archiven und Bibliotheken Südböhmens, nach Wilhelm Altmann neubearb. v. Petr Elbel, Přemysl Bar, Stanislav Bárta, und 
Sie sind grundsätzlich in den Ordensbriefregistern erhalten, welche zuletzt Jürgen Sarnowsky, Sebastian Kubon und Annika-Souhr Könighaus veröffentlicht haben. Ihre Bände umfassen bislang die Briefe bis zum Jahr 14 I $8 .{ }^{25}$ Die gedruckten Regesten aus den Ordensbriefregistern umfassen also bislang nicht das Ende der Regierung des Hochmeisters Michael Küchmeister sowie die gesamte Zeit der Regierung Pauls von Rusdorf ( I 422-I 44I). Diese Lücke wird teilweise durch das virtuelle Preußische Urkundenbuch gefüllt, dessen Regesten teils auf alten Editionen, teils auf archivalischer Überlieferung beruhen. ${ }^{26}$ Weil die großzügige (und auch lobenswerte) editorische Unternehmung als Work-in-progress Projekt konzipiert wird, muss man immer mit gewisser Unvollständigkeit rechnen. ${ }^{27}$

$\mathrm{Zu}$ der lückenhaften Materialaufnahme gesellt sich noch die Problematik einer Begriffsdefinition oder -abgrenzung hinsichtlich einer Gesandtschaft. Klaus

Lukáš Reitinger (Wien-Köln-Weimar: Böhlau, 2016). Für das hier behandelte Thema wäre es jedoch nützlicher, wenn der Band aus dem Hochmeistersarchiv entstanden wäre. Für den vorangegangenen Zeitraum muss man ältere Editionen mit dem diplomatischen Material, wie z.B. Codex diplomaticus Prussicus. Urkundensammlung zur älteren Geschichte Preussens aus dem königl. Geheimen Archiv zu Königsberg nebst Regesten, Bd. IV, hrsg. v. Johannes Voigt (Königsberg: Bei den Gebrüdern Bornträger, I 857); Bd. V, hrsg. v. Johannes Voigt (Königsberg: In Commission bei Wilhelm Koch, I 857 ); Bd. VI, hrsg. v. Johannes Voigt (Königsberg: In Commission bei Wilhelm Koch, I 86I), oder archivalische Überlieferung benutzen.

25 Regesten zu den Briefregistern des Deutschen Ordens: Die Ordensfolianten 2a, zaa und Zusatzmaterial. Mit einem Nachdruck von Kurt Lukas: Das Registerwesen der Hochmeister des deutschen Ritterordens, maschinenschrift. phil. Diss. Königsberg I92I (weiterhin als: RBDO I), hrsg. und bearb. v. Sebastian Kubon und Jürgen Sarnowsky, Beihefte zum Preußischen Urkundenbuch I (Göttingen: V\&R unipress, 201 2); Regesten zu den Briefregistern des Deutschen Ordens II: Die Ordensfolianten 8, 9 und Zusatzmaterial. Mit einem Anhang: Die Abschriften aus den Briefregistern des Folianten $A P G_{300,} / L I, 74$ (weiterhin als: RBDO II), hrsg. und bearb. v. Sebastian Kubon, Jürgen Sarnowsky, und Annika Souhr-Könighaus, Beihefte zum Preußischen Urkundenbuch 2 (Göttingen: V\&R unipress, 2014); Regesten zu den Briefregistern des Deutschen Ordens III: Die Ordensfolianten 5, 6 und Zusatzmaterial (weiterhin als: RBDO III), hrsg. und bearb. v. Jürgen Sarnowsky, Beihefte zum Preußischen Urkundenbuch 4 (Göttingen: V\&R unipress, 2017).

26 Virtuelles Preußisches Urkundenbuch (weiterhin als: VPU), bearb. v. Jürgen Sarnowsky, zugegriffen am I I. März 202 I, https://www.spaetmittelalter.uni-hamburg.de/Urkundenbuch/.

27 Als Beispiel dafür kann den in GStA PK aufbewahrte und aus der Regierungszeit des Hochmeisters Paul von Rusdorf stammende Ordensfoliant I 3 dienen, der in VPU bislang unvollständig erschlossen worden ist. Anhand meiner Recherchen zu diesem Ordensfolianten ist festzustellen, dass er um I 6 Briefe oder Schriftstücke des Hochmeisters an Kaiser Sigismund oder dessen Höflinge (wie z.B. Kaspar Schlick) umfasst, die jedoch in VPU noch nicht erschlossen werden. Allgemein zu Briefen der Hochmeister siehe Hartmut Boockmann, "Die Briefe des Deutschordenshochmeisters," in Kommunikationspraxis und Korrespondenzwesen im Mittelalter und in der Renaissance, hrsg. v. Heinz-Dieter Heimann in Verbindung mit Ivan Hlaváček (PaderbornWien-München-Wien-Zürich: Ferdinand Schöningh, I 998), I 03 - I 2. 
Neitmann unterscheidet zwei Gesandtschaftstypen. Erstens, wenn „ein Herrscher seine Gesandtschaften in unregelmäßigen Abständen je nach den politischen Erfordernissen zu Verhandlungen über anstehende Probleme in andere Länder schickt," und zweitens, wenn „zwei oder mehrere Herrscher ihre Gesandtschaften zu gemeinsamen Beratungen an einen bestimmten Grenzpunkt entsenden. ${ }^{\text {“28 }}$ Den letzteren Gesandtschaftstyp darf man wohl außer Betracht lassen, wenn man darauf in den Quellen kaum stoßen kann. Dies kann wohl damit erklärt werden, dass die beiden Herrschaftsgebiete nicht unmittelbar aneinandergrenzten.

Obgleich die Gesandtschaften oder die Gesandten, die mit den entsprechenden diplomatischen Schriftstücken gereist sind, per definitionem und theoretisch ziemlich eindeutig von anderen Reisen und Reisenden (wie z.B. Briefboten) abgegrenzt werden können, verursacht jedoch die erwähnte unvollständige Überlieferung gewisse praktische Schwierigkeiten in der Identifikation der Gesandtschaften oder Gesandten in den Quellen.

Im „Marienburger Tresslerbuch“ wird zwar eine relativ klare Grenzlinie zwischen einem bevollmächtigten Gesandten und einem Briefboten gezogen, wenn die ersten meistens mit dem Namen des Gesandten und der Wendung in unsers homeysters botschaft (oder in unser homeyster ken Ungarn vorsante) erscheinen, während die letzteren als unbenannte boten mit unsers homeisters briefen angeführt wurden. ${ }^{29}$ Einige im Tresslerbuch vorkommende Einträge können jedoch nicht immer so deutlich beurteilt werden. Kann man ausschließen, dass das Abschicken von Falken oder Lachs nach Ungarn und umgekehrt der Weintransport aus Ungarn nach Preußen mit Gesandtschaften verbunden wurden?30

Peter Wargel, der Diener des Hochmeisters, unternahm (um I 405) beispielsweise eine Reise durch einige Länder (Ungarn, Österreich, Polen usw.), um dem Hochmeister Bericht über die politischen Verhältnisse in den jeweiligen Ländern zu erstatten. ${ }^{31}$ Im Jahre 1409 war er im Gefolge der Ordensgesandten (Werner von Tettingen, Oberster Spittler, und Albrecht Graf von Schwarzburg, Komtur von Thorn), die zu König Sigismund nach Kaschau zogen, und fungierte als Verbindungsperson zwischen der sich nähernden Gesandtschaft und dem sich in Kaschau aufhaltenden königlichen Hof. ${ }^{32}$ Dieselbe Rolle spielte er auch während des Konzils zwischen den in Konstanz bleibenden Ordensgesandten und dem

28 Neitmann, Die Staatsverträge, I 5.

29 Das Marienburger Tresslerbuch, hrsg. v. Joachim, 344, 467, 488, 560.

30 Ebd., 383, 386, 423-424, 478, 526, 538, 593.

3 I GStA PK, XX. HA, OBA, Nr. 838 .

32 GStA PK, XX. HA, OBA, Nr. I 175. 
Hof Sigismunds, der quer durch Westeuropa reiste. ${ }^{33}$ Gerade ihm wurde anvertraut, dem römischen König die Wahlanzeige des neuen Hochmeisters (Michael Küchmeister) zu überbringen. ${ }^{34}$ Sigismund verteidigte ihn sogar gegen bestimmte Vorwürfe seitens des Hochmeisters und empfahl ihm ihn. ${ }^{35}$ War Peter Wargel nur ein schlichter Briefbote oder jedoch noch etwas mehr?36

Eine Grenzziehung zwischen den Gesandtschaften und den Briefboten (schließlich sind diese fast hinter jeden Brief zu vermuten) ist jedoch nicht die einzige unverzichtbare Aufgabe. Man muss auch - soweit die Quellen es ermöglichen - die Angelegenheiten beurteilen, welche die Delegationen zu erledigen versuchten, ob diese die ihres Auftraggebers oder ihre eigenen waren. Diese beiden Bereiche konnten sich überschneiden. Beispielweise forderte der Ofener Bürger Hans Stadler 15000 ung. Gulden vom Hochmeister aufgrund einer königlichen Urkunde (vom 6. Februar I 4I 2) in seiner Hand. Bei diesem Betrag handelte es sich um eine Schuldsumme des Ordens gegenüber König Sigismund, der sich sie von Hans Stadler auszahlen ließ und die Schuld des Hochmeisters an ihn überwies. ${ }^{37}$ Mit Rücksicht auf die Überlieferungssituation kann ein solcher Aspekt bei den Ordensgesandtschaften besser erforscht werden. Bei den königlichen Delegationen muss demgegenüber der breitere Kontext beachtet und kann dementsprechend nicht jedes königliche Empfehlungsschreiben als Nachweis einer bevollmächtigten Gesandtschaft betrachtet werden. ${ }^{38}$

33 Die Berichte der Generalprokuratoren des Deutschen Ordens an der Kurie, Bd. II, Peter von Wormditt (I403-I4I9), bearb. v. Hans Koeppen, Veröffentlichungen aus den Archiven Preußischer Kulturbesitz I 3 (Göttingen: Vandenhoecken \& Ruprecht, I 960), passim.

34 GStA PK, XX. HA, OBA, 204I/2042; RI, XI, I-2, hrsg. v. Altmann, Nr. 967; RBDO II, hrsg. und bearb. v. Kubon, Sarnowsky, und Souhr-Könighaus, 266 Nr. 8.

35 GStA PK, XX. HA, OBA, Nr. 2360 ; RI, XI, I-2, hrsg. v. Altmann, Nr. 1964.

36 Am Ende des königlichen Briefs (siehe Anm. 34) wird Laurencius vom König dem Hochmeister empfohlen. War er auch nicht nur der schlichte Briefbote, der mit einer mündlichen Botschaft ausgestattet wurde (siehe GStA PK, XX. HA, OBA, Nr. 204I/2042: Laurencius unser diener und lieber getruer entworter diss briefs von unsern wegen sagen wirdt, das wollest im genczlich gelouben)?

37 RI, XI, I-2, hrsg. v. Altmann, Nr. I 88; Markian Pelech, "Quellen über den Hochmeister Heinrich von Plauen (1410-1413) im Bayerischen Hauptstaatsarchiv in München und in Wien," Zeitschrift für Ostforschung 34, H. 2 (1985): 26-27.

38 Das private Interesse verbarg sicher hinter der Reise (1 42 I ) des königlichen Arztes Sigfried Degenberg, der versuchte, eine Pfründe zu Frauenburg in Preußen mithilfe des Empfehlungsschreibens Sigismunds vor Hochmeister in Marienburg zu erlangen, siehe: RI, XI, I-2, hrsg. v. Altmann, Nrn. 4600, 4656, 4679. Zu Degenberg siehe Michalina Broda, Lekarze w państwie zakonu krzyżackiego $w$ Prusach $w X I V-X V$ wieku (Kraków: Universitas, 2013), 193-194; und Miriam Hlavačková, "Lekár troch král'ov. Bratislavský a vyšehradský prepošt Siegfried Degenberg a jeho zdravotné rady," Studia Historica Tyrnaviensia I 6 (2014): 260-266. 
Diese Vorbehalte betreffen vor allem diejenigen Vorfälle, die nur von fragmentarischer Überlieferung beleuchtet werden können. Wo diplomatische Schriftstücke (besonders Berichte) und Briefe vorhanden sind, kann man den gesamten Ablauf einer Gesandtschaft bis auf die Einzelheiten analysieren. Die Berichte der Ordensgesandtschaften beinhalten meistens den Zeitablauf (die Ankunft, den Wegzug, manchmal die Reise selbst), die Darstellung der Verhandlung mit dem König bzw. dessen Höflingen einschließlich der direkten Reden der Beteiligten und gelegentlich die eigenen Ratschläge für den Hochmeister. Diesbezüglich sind einige Gesandtschaften besonders erwähnenswert.

Das erste Beispiel fällt in den Frühling I 4 I 3, als der Hochmeister Heinrich von Plauen seine Gesandten nach Italien (Udine) entsandte. Ihr Vorhaben war es, die Beschwerden des Hochmeisters über die Tätigkeit des königlichen Juristen Benedict Makrai dem römischen König vorzubringen. ${ }^{39} \mathrm{Zu}$ den bedeutenden Ordensgesandtschaften sind jeweils mehr als ein Bericht überliefert. Über die Verhandlungen in Ofen ab Mai bis Juni I 4 I 4 ließ der Gesandte und Kumpan des Hochmeisters, Jost von Hohenkirchen, fünf Berichte verfassen! ${ }^{+\circ}$ Demgegenüber verschickten Martin Kemnate, Oberster Marschall, und der Gesandte nach Breslau (1419/1 420), „nur“ drei Berichte, von denen der erste in Thorn noch vor dem Aufbruch der Gesandtschaft entstanden war. ${ }^{41}$ Eine Reihe sehr merkwürdiger Berichte sind vom Ordensbruder Nikolaus von Redewitz erhalten, der vom Hochmeister auf Drängen Sigismunds im Jahr 1427 nach Ungarn entsandt wurde, um den Plan einer Ansiedlung von Ordensbrüdern in Ungarn zu besprechen und zu realisieren..$^{42}$

Manchmal ermöglichen weder ausführliche Berichte noch eine Menge der anderen erhaltenen Quellen, die Gesandtschaften in genannter Weise einzuordnen

39 GStA PK, XX. HA, OBA, Nr. 1935. Der beachtenswerte Bericht wird bei Wiesław Sieradzan, Misja Benedykta Makraia w latach I4I2-I4I3. Z dziejów pokojowych metod rozwiazywania konfliktów międzypaństwowych w Europie Środkowo-Wschodniej w póżnym średniowieczu (Malbork: Muzeum Zamkowe, 2009), I69-178, gedruckt, jedoch sehr mangelhaft.

40 GStA PK, XX. HA, OBA, Nrn. 2073 (I 4. Mai [1 4I 4], Ofen), 2076 (20. Mai I 4I 4, Ofen), 2077 (20. Mai I 4 I 4, Ofen), 2078 (20. Mai I 4 I 4, Ofen), 2079 (20. Mai I 4 I 4 ).

41 GStA PK, XX. HA, OBA, Nrn. 3059 (14. Dezember 1419, Thorn), 3060 (20. Dezember I 419), 3083; Deutsche Reichstagsakten, Bd. VII, Unter Kaiser Sigmund, I. Abt., I4IO-1420, hrsg. v. Dietrich Kerler (München: Rudolph Oldenbourg, I 878), $405-406$ Nr. 278 (7. Januar [1 420$]$, Breslau).

42 Erich Joachim, "König Sigmund und der Deutsche Ritterorden in Ungarn, I 429-I 432 (Mitteilungen aus dem Staatsarchiv zu Königsberg)," Mitteilungen des Instituts für Österreichische Geschichtsforschung 33, H. I ( I 9 I 2): 87-I I 9; vgl. Gerhardt Hochstrasser, "Der Ordenspraeceptor Nicolaus von Redewitz und die Münzprägung in Siebenbürgen und im Szeweriner Banat im I 5. Jahrhundert," in Beiträge zur Geschichte des Deutschen Ordens, Bd. 2, hrsg. v. Udo Arnold (Marburg/Lahn: Elwert, I 993), I 24-I 34. 
oder sogar zu bestimmen, ob man mit einer oder mehreren Gesandtschaften zu tun hat. Das ist für die Gesandtschaft bzw. die Gesandtschaften zum Konzil von Konstanz, wo die Gesandten unter anderem auch mit König Sigismund verhandeln sollten, der Fall. ${ }^{43}$

Die entsprechende Vollmacht des Hochmeisters Michael Küchmeister für die Ordensgesandten wurde am I 4. Oktober I 4 I 4 ausgestellt. ${ }^{44}$ Diese bildeten jedoch zum Zeitpunkt der Ausstellung jener Vollmacht faktisch keine einheitliche Delegation. Jeder von ihnen hielt sich an einem anderen Ort auf und nur einige machten sich direkt von Preußen aus auf den Weg nach Konstanz.

Noch schwieriger ist das Ende, bzw. die Rückkehr der Gesandtschaft festzustellen. Während des Konstanzer Konzils herrschte nämlich eine relativ hohe Fluktuation der Gesandten. Einige verließen die Stadt ziemlich bald, ohne durch jemanden ersetzt zu werden. Kurz nachdem sich König Sigismund zu einer Diplomatenreise durch Westeuropa aufgemacht hatte, verließ auch der Komtur von Christburg Friedrich von Welden Konstanz in Richtung Preußen (wo er übrigens kurz danach starb). Seine Rolle auf dem Konzil wurde vom Komtur von Thorn Johann von Selbach übernommen, der frühestens im September I 4 I 5 am Bodensee eintraf und ein aktives Mitglied der Ordensdelegation wurde.

In Konstanz wurden immer wieder Stimmen laut (auch seitens König Sigismunds), dass der Orden nicht ausreichend auf dem Konzil vertreten sei und dass die Zusammensetzung der Ordensgesandtschaft der Wichtigkeit seiner Mission nicht entspreche. Die Ordensgesandten erlaubten sich deswegen dem Hochmeister sogar konkrete Namen zu empfehlen. Die Ordensdelegation einigte sich darauf, dass es höchst wünschenswert sei, noch den Danziger (später Elbinger) Komtur Heinrich Holt zu entsenden, der zur engeren Umgebung König Sigismunds gehörte, der sich angeblich die Ansichten des genannten Komturs bereitwillig anhörte. ${ }^{45}$

43 Die folgenden Abschnitte beruhen grundsätzlich auf Přemysl Bar, Diplomacie, právo a propaganda v pozdnim středověku. Polsko-litevská unie a Řád nèmeckých rytîrù na kostnickém koncilu (I4I4-I4I8) (Brno: Filozofická fakulta, Masarykova univerzita, 2017), 42-8I, wo einzelne Quellenhinweise zu finden sind; vgl. ders., "Eine (un)genutzte Gelegenheit? Die Polnischlitauische Union und der Deutsche Orden auf dem Konstanzer Konzil ( 14 I 4-1 4 I 8)," in Der Deutsche Orden auf dem Konstanzer Konzil. Pläne - Strategien - Erwartungen, hrsg. v. Helmut Flachenecker unter Mitarbeit von Tobias Baus und Katharina Kemmer, Quellen und Studien zur Geschichte des Deutschen Ordens 84 (Ilmtal-Weinstraße: VDG, 2020), 35-54.

${ }_{44}$ GStA PK, XX. HA, Pergamenturkunden, Schiebl. 65, Nr. 3; Die Berichte der Generalprokuratoren, II, bearb. v. Koeppen, Nr. i I o; RBDO II, hrsg. und bearb. v. Kubon, Sarnowsky, und Souhr-Könighaus, I $23-$ I 24 Nr. 85.

45 Der Brief des Ordensprokurators vom 6. Januar I 4 I 7 (Die Berichte der Generalprokuratoren, II, bearb. v. Koeppen, 380 Nr. I 92): Der Ordensdelegation halte es einmütig für gut, den Komtur von Danzig nach Konstanz zu entsenden, weil er dem römischen König wol bekant sei, und 
Hochmeister Michael Küchmeister nahm sich jene Empfehlung zumindest teilweise zu Herzen, verschob jedoch die Komplettierung der Delegation offenbar bis zum März I 417, weil König Sigismund sich bis dahin nicht in Konstanz befand. Im Empfehlungsschreiben an den römischen König ernannte der Hochmeister drei Ordensgebietiger: den Elbinger Komtur und Obersten Spittler Heinrich Holt, den Komtur von Mewe und Obersten Trappier Johann von Selbach (den ehemaligen Komtur von Thorn) sowie den Komtur von Balga Ulrich Zenger. Diese Gebietiger schlossen sich der bestehenden Konstanzer Delegation an und bildeten seitdem gemeinsam eine Ordensgesandtschaft.

Diese Darstellung der Genese der Konstanzer Ordensdelegation wirft die Frage auf, ob man von einer oder zwei Gesandtschaften nach Konstanz sprechen soll (eine vom Oktober I 4 I 4 und eine andere vom März I 4 I 7). Eine präzise Abgrenzung ist vielleicht nicht möglich, wenn man dazu noch in Betracht zieht, dass einzelne Gesandte während des Konzils mehrmals hin und zurück reisten. Weiter steht die Zusammenstellung der Konstanzer Gesandtschaft im Zusammenhang mit anderer wichtiger Problematik: welche Personen wurden in die Gesandtschaften aufgenommen und nach welchen Kriterien wurden sie ausgewählt?

Obwohl der konkrete Ablauf der Wahl von Personen in den Quellen nicht nachverfolgt werden kann, scheint die These Klaus Neitmanns, die Gesandten seien nicht aufgrund der von ihnen wahrgenommenen Ämter, sondern vielmehr aufgrund ihrer persönlichen Fähigkeiten und Kompetenzen ausgewählt worden, richtig zu sein. ${ }^{46}$

Diese These könnte, zumindest teilweise, auch für die Wahl der Gesandten seitens König Sigismunds gelten. Man muss jedoch davon ausgehen, dass er jeweils aktuell auf bestimmte Situationen reagierte und aus den sich gerade an seinem Hof befindlichen Höflingen diejenigen Personen auswählte, die ihm die fähigsten und kompetentesten zu sein schienen. ${ }^{77}$ Dies war jedoch nicht immer ausreichend, wie es die oben genannte, im durch Spannungen geprägten Sommer I 49 nach Preußen entsandte Gesandtschaft zeigt (vgl. Anm. 23). Sigismund schickte damals Herzog Přemek von Troppau und seinen eigenen Hofmeister, Ludwig von Ottingen, mit der Aufgabe nach Preußen, den Hochmeister dazu zu bewegen, Sigismund als Schiedsrichter im Streit mit Polen zu akzeptieren. Beide Gesandten trafen im Juni in Thorn ein, wo sie persönlich mit dem Hochmeister verhandelten, aber ihre Aufgabe nicht erledigten. Erst die nachfolgende königliche Gesandtschaft unter

vormag in, also das her syns willen bas von im undirwiset mochte werden denne ymands anders, und mochte im ouch myt ernstlicher ermanunge czusprechen denn eyn ander.

46 Neitmann, Die Staatsverträge, 7 I -75.

47 Man sollte auch nicht ausschließen, dass die Wahl manchmal bloße Zufälligkeit sein konnte. 
der Leitung des Erzbischofs Bartholomeus von Mailand war im Stande den Hochmeister zu einem Kurswechsel zu veranlassen und ihn sogar mit dem König von Polen zu versöhnen, wenn auch nur vorübergehend. $4^{8}$

Höchstwahrscheinlich war König Sigismund zutiefst überzeugt, sogenannte "doppelte Gesandte“ seien für den wechselseitigen diplomatischen Verkehr am besten geeignet. Zumindest äußerte er sich entsprechend über den Ordensbruder Nikolaus von Redewitz, der sowohl ihm als auch dem Hochmeister als Gesandter diente. ${ }^{49}$ Sehr hoch schätzte der König auch den Rigaer (später Lütticher) Erzbischof Johann von Wallenrode. Er war ein Ordensbruder, der seine Karriere als Diplomat schon am Hof König Ruprechts von der Pfalz begonnen hatte. Wallenrodes diplomatische Tätigkeit war tatsächlich dem römischen König viel nützlicher als dem Hochmeister. Während des Konstanzer Konzils wollte Michael Küchmeister den Erzbischof sogar wegen übertriebener finanzieller Forderungen als Ordensgesandten abberufen, wobei er auch behauptete, Wallenrode vertrete die Interessen des römischen Königs viel stärker als jene des Ordens. Der Ordensprokurator riet dem Hochmeister jedoch von einem solchen Schritt dezidiert und mit Erfolg ab. ${ }^{5 \circ}$

Die beiden genannten Ordensbrüder, Nikolaus von Redewitz und Johann von Wallenrode, wurden auch zu königlichen Räten ernannt. Die Frage, ob es bei ähnlichen Fällen, also bei Gesandten, die beiden Herrschern gleichzeitig dienten, immer zu solchen Ratsernennungen kam, muss noch offenbleiben. Für zwei weitere Personen, bei welchen man ein solches Vorgehen vermuten könnte, fehlen konkrete Quellenbelege. Es handelt sich um die Komture Heinrich Holt und Ludwig von Landsee, die mehrmals von beiden Seiten mit diplomatischen Missionen beauftragt wurden. Ludwig von Landsee, der wohl bekannteste Ordensdiplomat,

48 Vgl. Nowak, Międzynarodowe procesy, 86-88.

49 Siehe den Brief König Sigismunds an den Hochmeister Paul von Rusdorf vom 9. April I 427 (GStA PK, XX. HA, OBA, Nr. 4738; RI, XI, I-2, hrsg. v. Altmann, Nr. 6887): [...] uns ist wol $z u$ dank, daz du uns den ersamen Niklas Redwicz bruder dines Ordens gesandt hast, und ist fast für dich und dinen orden, daz wir allzit ewers ordens einen oder zwen bi uns und in unserm rate haben, dann understunden soliche sachen an uns langen, dorzu wir des ordens wol bedurfen und davon dem orden ere und frome wachßen mogen. und doruf haben wir uns den vorgenant Redwicz in unsern rate lassen sweren [...].

so Bernhart Jähnig, Johann von Wallenrode O. T. Erzbischof von Riga, Königlicher Rat, Deutschordensdiplomat und Bischof von Lüttich im Zeitalter des Schismas und des Konstanzer Konzils (um 1370-1419), Quellen und Studien zur Geschichte des Deutschen Ordens 24 (Bad Godesberg: Verl. Wiss. Archiv, I 970); ders., "Johannes von Wallenrode und das Konstanzer Konzil," in Der Deutsche Orden, hrsg. v. Flachenecker unter Mitarbeit v. Baus und Kemmer, I07-I 25. 
verhandelte in den Jahren I 420,51 I 422 und I 423 mehrmals wochenlang mit König Sigismund. Trotzdem gibt es, soweit mir bekannt ist, kein Quellenzeugnis über seine Ernennung zum königlichen Rat (vgl. Anm. I9).

Abschließend muss man wieder unterstreichen, dass Klaus Neitmanns Thesen über die Ordensgesandtschaften nach wie vor nützlich und eine unentbehrliche Ausgangbasis für weitere Forschungen sind. Die Behandlung dieser Gesandtschaften aus der exklusiven Perspektive des Hochmeisters bzw. des Ordens scheint jedoch zu beschränkt zu sein. Die wechselseitige Perspektive ermöglicht eine bessere Abgrenzung einzelner Gesandtschaften und kann zum Verständnis der Mechanismen beitragen, die hinter der Wahl der Gesandten standen. Nicht zuletzt ist darauf hinzuweisen, dass einige einzigartige Quellen wie Instruktionen und Berichte, die im Ordensbriefarchiv zu einigen Gesandtschaften zwischen Hochmeister und Kaiser Sigismund überliefert sind, noch nicht ausreichend von der Forschung ausgewertet worden sind, obgleich sie die hier interessierenden Beziehungen wesentlich beleuchten könnten. Diese und die anderen hier nicht behandelten Fragen wird man erst nach einer ausführlichen und vollständigen $\mathrm{Zu}$ sammenstellung aller Gesandtschaften beantworten können.

\section{PRIMARY SOURCES:}

Berlin. Geheimes Staatsarchiv Preußischer Kulturbesitz. XX. Hauptabteilung, Ordensbriefarchiv, Nrn. 838, I I 75, I 935, 204I/2042, 2073, 2076, 2077, 2078, 2079, 2360, 2977, 3059, 5060, 4083, 3174, 4738.

Berlin. Geheimes Staatsarchiv Preußischer Kulturbesitz. XX. Hauptabteilung, Pergamenturkunden, Schiebl. 65, Nr. 3.

Codex diplomaticus Prussicus. Urkundensammlung zur älteren Geschichte Preussens aus dem königl. Geheimen Archiv zu Königsberg nebst Regesten. Bd. IV. Herausgegeben von Johannes Voigt. Königsberg: Bei den Gebrüdern Bornträger, I 857.

Codex diplomaticus Prussicus. Urkundensammlung zur älteren Geschichte Preussens aus dem königl. Geheimen Archiv zu Königsberg nebst Regesten. Bd. V. Herausgegeben von Johannes Voigt. Königsberg: In Commission bei Wilhelm Koch, I 857.

Codex diplomaticus Prussicus. Urkundensammlung zur älteren Geschichte Preussens aus dem königl. Geheimen Archiv zu Königsberg nebst Regesten. Bd. VI. Herausgegeben von Johannes Voigt. Königsberg: In Commission bei Wilhelm Koch, I 86 I.

Das Marienburger Tresslerbuch der Jahre 1399-1409. Herausgegeben von Erich Joachim. Königsberg i. Pr.: Thomas \& Oppermann, 1896.

5I Zu dieser Gesandtschaft ist ein beachtenswerter Bericht erhalten (30. Mai I 420), weil der Komtur gemeinsam mit dem königlichen Heer in hussitisches Böhmen eintraf, siehe GStA PK, XX. HA, OBA, Nr. 3174 . 
Deutsche Reichstagsakten. Bd. VII. Unter Kaiser Sigmund. I. Abt. I4IO-I420. Herausgegeben von Dietrich Kerler. München: Rudolph Oldenbourg, I 878.

Die Berichte der Generalprokuratoren des Deutschen Ordens an der Kurie. Bd. II. Peter von Wormditt (I403-I4I9). Veröffentlichungen aus den Archiven Preußischer Kulturbesitz I 3. Bearbeitung von Hans Koeppen. Göttingen: Vandenhoecken \& Ruprecht, I 960.

Die Staatsverträge des Deutschen Ordens in Preussen im Is. Jahrhundert. Bd. I (1398-I437). Herausgegeben von Erich Weise. Marburg: N. G. Elwert, I970.

Regesta Imperii, XI. Die Urkunden Kaiser Sigmunds (I4IO-I437). Bd. I-2 (I4IO-I437). Herausgegeben von Wilhelm Altmann. Innsbruck: Verlag der Wagner'schen Universitäts-Buchhandlung, I 896-1 900.

Regesta Imperii, XI. Regesten Kaiser Sigismunds (14I0-1437) nach Archiven und Bibliotheken geordnet. Bd. I. Die Urkunden und Briefe aus den Archiven und Bibliotheken Mäbrens und Tschechisch-Schlesiens. Nach Wilhelm Altmann neubearbeitet von Peter Elbel. Wien-Köln-Weimar: Böhlau, 20 I 2.

Regesta Imperii, XI. Regesten Kaiser Sigismunds (14I0-1437) nach Archiven und Bibliotheken geordnet. Bd. 2. Die Urkunden und Briefe aus den Archiven und Bibliotheken West-, Nord-und Ostböhmens. Nach Wilhelm Altmann neubearbeitet von Petr Elbel, Stanislav Bárta, Přemysl Bar, Lukáš Reitinger. Wien-Köln-Weimar: Böhlau Verlag, 2015 .

Regesta Imperii, XI. Regesten Kaiser Sigismunds (14I0-1437) nach Archiven und Bibliotheken geordnet. Bd. 3. Die Urkunden aus den Archiven und Bibliotheken Südböhmens. Nach Wilhelm Altmann neubearbeitet von Petr Elbel, Přemysl Bar, Stanislav Bárta, Lukáš Reitinger. Wien-Köln-Weimar: Böhlau, 20 I 6.

Regesten zu den Briefregistern des Deutschen Ordens: Die Ordensfolianten 2a, 2aa und Zusatzmaterial. Mit einem Nachdruck von Kurt Lukas: Das Registerwesen der Hochmeister des deutschen Ritterordens, maschinenschrift. phil. Diss. Königsberg Ig2I. Herausgegeben und bearbeitet von Sebastian Kubon und Jürgen Sarnowsky. Beihefte zum Preußischen Urkundenbuch I. Göttingen: V\&R unipress, 20 I 2.

Regesten zu den Briefregistern des Deutschen Ordens II: Die Ordensfolianten 8, 9 und Zusatzmaterial. Mit einem Anhang: Die Abschriften aus den Briefregistern des Folianten $A P G$ 300, R/LI, 74. Herausgegeben und bearbeitet von Sebastian Kubon, Jürgen Sarnowsky, und Annika Souhr-Könighaus. Beihefte zum Preußischen Urkundenbuch 2. Göttingen: V\&R unipress, 20 I 4.

Regesten zu den Briefregistern des Deutschen Ordens III: Die Ordensfolianten 5, 6 und Zusatzmaterial. Herausgegeben und bearbeitet von Jürgen Sarnowsky. Beihefte zum Preußischen Urkundenbuch 4. Göttingen: V\&R unipress, 20 I 7.

\section{SECONDARY SOURCES:}

Bar, Přemysl. "A Tortuous Path to Reconciliation and Justice. Sigismund of Luxembourg as Arbiter in the Dispute between the Teutonic Knights and Poland ( $1412-1420) . "$ Zeitschrift für Ostmitteleuropa-Forschung 66 (2017): 3-40. 
Bar, Přemysl. “Der 'Krönungssturm’ König Sigismund von Luxemburg, Großfürst Witold von Litauen und das gescheiterte politische Bündnis zwischen beiden Herrschern.” Roczniki Historyczne 83 (2017): 65-1 01 .

Bar, Přemysl. Diplomacie, právo a propaganda v pozdním středověku. Polsko-litevská unie a ̌̌ád nèmeckých rytírú na kostnickém koncilu (I4I4-I4I8). Brno: Filozofická fakulta, Masarykova univerzita, 2017.

Bar, Přemysl. "Eine (un)genutzte Gelegenheit? Die Polnisch-litauische Union und der Deutsche Orden auf dem Konstanzer Konzil ( I 4 I 4- I 4 I 8)." In Der Deutsche Orden auf dem Konstanzer Konzil. Pläne - Strategien - Erwartungen, herausgegeben von Helmut Flachenecker unter Mitarbeit von Tobias Baus und Katharina Kemmer, 35-54. Quellen und Studien zur Geschichte des Deutschen Ordens 84. Ilmtal-Weinstraße: VDG, 2020.

Boockmann, Hartmut. "Die Briefe des Deutschordenshochmeisters." In Kommunikationspraxis und Korrespondenzwesen im Mittelalter und in der Renaissance, herausgegeben von Heinz-Dieter Heimann in Verbindung mit Iva Hlaváček, I03- I I 2. Paderborn-Wien-München-Wien-Zürich: Ferdinand Schöningh, I 998.

Boockmann, Hartmut. "Nachrichten über die Diplomatie des Deutschen Ordens aus dem 'Tresslerbuch'." In Balticum. Studia z dziejów polityki, gospodarki i kultury XII-XVII wieku ofiarowane Marianowi Biskupowi w siedemdziesiata rocznice urodzin, herausgegeben von Zenon Hubert Nowak, 67-78. Toruń: Towarzystwo Naukowe w Toruniu, 1992.

Broda, Michalina. Lekarze w państwie zakonu krzyżackiego w Prusach w XIV-XV wieku. Kraków: Universitas, 2013.

Hlavačková, Miriam. "Lekár troch král'ov. Bratislavský a vyšehradský prepošt Siegfried Degenberg a jeho zdravotné rady." Studia Historica Tyrnaviensia I 6 (2014): 260-266.

Hochstrasser, Gerhardt. "Der Ordenspraeceptor Nicolaus von Redewitz und die Münzprägung in Siebenbürgen und im Szeweriner Banat im I s. Jahrhundert.” In Beiträge zur Geschichte des Deutschen Ordens. Bd. 2, herausgegeben von Udo Arnold, I 24- I 34. Marburg/Lahn: Elwert, I 993.

Hoensch, Jörg K. “König/Kaiser Sigismund, der Deutsche Orden und Polen-Litauen. Stationen einer problembeladenen Beziehung." Zeitschrift für Ostmitteleuropa-Forschung 46 ( I 997): I-44.

Israel, Ottokar. Das Verhältnis des Hochmeisters des Deutschen Ordens zum Reich im I5. Jahrhundert. Marburg: Johann Gottfried Herder Institut, I 952.

Jähnig, Bernhart. Johann von Wallenrode O. T. Erzbischof von Riga, Königlicher Rat, Deutschordensdiplomat und Bischof von Lüttich im Zeitalter des Schismas und des Konstanzer Konzils (um 1370-I4I9). Quellen und Studien zur Geschichte des Deutschen Ordens 24. Bad Godesberg: Verl. Wiss. Archiv, 1970.

Jähnig, Bernhart. "Johannes von Wallenrode und das Konstanzer Konzil." In Der Deutsche Orden auf dem Konstanzer Konzil. Pläne - Strategien - Erwartungen, herausgegeben von Helmut Flachenecker unter Mitarbeit von Tobias Baus und Katharina 
Kemmer, I07-I 25. Quellen und Studien zur Geschichte des Deutschen Ordens 84. Ilmtal-Weinstraße: VDG, 2020.

Joachim, Erich. "König Sigmund und der Deutsche Ritterorden in Ungarn, I 429- I 432 (Mitteilungen aus dem Staatsarchiv zu Königsberg)." Mitteilungen des Instituts für Österreichische Geschichtsforschung 33, H. I ( I 9 I 2): 87- I I 9.

Kintzinger, Martin. Westbindungen im spätmittelalterlichen Europa. Auswärtige Politik zwischen dem Reich, Frankreich, Burgund und England in der Regierungszeit Kaiser Sigmunds. Stuttgart: Thorbecke, 2000.

Lückerath, Carl A.. Paul von Rusdorf. Hochmeister des Deutschen Ordens, I422-I44I. Quellen und Studien zur Geschichte des Deutschen Ordens i s. Bad Godesberg: Verlag Wissenschaftliches Archiv, 1969.

Matison, Ingrid. "Die Lehnsexemtion des Deutschen Ordens und dessen staatsrechtliche Stellung in Preußen." Deutsches Archiv für Erforschung des Mittelalters 2 I (1965): I 94-248.

Neitmann, Klaus. Die Staatsverträge des Deutschen Ordens in Preußen I230-I449. Studien zur Diplomatie eines spätmittelalterlichen deutschen Territorialstaates. Köln-Wien: Böhlau Verlag, I 986.

Neitmann, Klaus. "Ludwig von Landsee, ein Gebietiger des Deutschen Ordens in Preußen im I 5. Jahrhundert. Beobachtungen zur Außenpolitik des Ordens.” Jabrbücher für Geschichte Osteuropas N.F. 36 ( I 988): I 6 I - 190.

Nikodem, Jarosław. Polska i Litwa wobec husyckich Czech w latach 1420-1433. Studium o polityce dynastycznej Wtadystawa Jagietty i Witolda Kiejstutowicza. Oświęcim: Napoleon V, 2015,2 . Auflage.

Nowak, Zenon H.. Miedzynarodowe procesy polubowne jako narzędzie polityki Zygmunta Luksemburskiego w pótnocnej i środkowowschodniej Europie (I4I2-I424). Toruń: Uniwersytet Mikołaja Kopernika, I 98 I.

Nowak, Zenon H.. Polityka pótnocna Zygmunta Luksemburskiego do roku I4II. Roczniki Towarzystwa Naukowego w Toruniu 69/r. Toruń: Państwowe Wydawnictwo Naukowe, i 964.

Nöbel, Wilhelm. Michael Küchmeister. Hochmeister des Deutschen Ordens I4I4-I422. Quellen und Studien zur Geschichte des Deutschen Ordens 5. Bad Godesberg: Verlag Wissenschaftliches Archiv, 1969.

Pelech, Markian. "Quellen über den Hochmeister Heinrich von Plauen ( I 4 IO-I 4 I 3 ) im Bayerischen Hauptstaatsarchiv in München und in Wien." Zeitschrift für Ostforschung 34, H. I (1985): 19-38.

Reitemeier, Arnd. Außenpolitik im Spätmittelalter. Die diplomatischen Beziehungen zwischen dem Reich und England 1377-1422. Paderborn-München-Wien-Zürich: Schöningh 1999 .

Sieradzan, Wiesław. Misja Benedykta Makraia w latach I4I2-I4I3. Z dziejów pokojowych metod rozwiązywania konfliktów międzypaństwowych w Europie Środkowo-Wschodniej w póżnym średniowieczu. Malbork: Muzeum Zamkowe, 2009. 